\title{
Altered brain activation to colorectal distention in visceral hypersensitive maternal-separated rats
}

\author{
M. M. WOUTERS, ${ }^{\star}$ S. VAN WANROOY, ${ }^{\star}$ C. CASTEEls $, \dagger, \dagger$ A. NEMETHOVA, ${ }^{\star}$ A. DE VRIES, ${ }^{\star}$ L. VAN OUDENHOVE, ${ }^{\star}$ \\ R. M. VAN DEN WIJNGAARD, $\S \mathrm{K}$. VAN LAERE $\dagger, \ddagger$ \&. BOECKXSTAENS * \\ ${ }^{\star}$ Division of Gastroenterology, TARGID, Translational Research Center for Gastrointestinal Disorders, Catholic University \\ of Leuven, Leuven, Belgium \\ $†$ MoSAIC, Molecular Small Animal Imaging Center, University of Leuven, Leuven, Belgium \\ \$Division of Nuclear Medicine, University Hospital Gasthuisberg, Leuven, Belgium \\ $\S$ Tytgat Institute for Liver and Intestinal Research, Academic Medical Center, Amsterdam, The Netherlands
}

\begin{abstract}
Background Early life trauma can predispose to increased visceral pain perception. Human neuroimaging studies emphasize that altered brain processing may contribute to increased visceral sensitivity. The aim of our study was to evaluate brain responses to painful visceral stimuli in maternal-separated rats before and after acute stress exposure in vivo. Methods $\mathrm{H}_{2}{ }^{15} \mathrm{O}$ microPET scanning was performed during colorectal distention in maternal-separated rats before and after water avoidance stress. Brain images were anatomically normalized to Paxinos space and analyzed by voxel-based statistical parametric mapping (SPM2). Colorectal induced visceral pain was assessed by recording of the visceromotor response using abdominal muscle electromyography. Key Results Colorectal distention (1.0-2.0 mL) evoked a volume-dependent increase in visceromotor response in maternal-separated rats. Stress [water avoidance (WA)] induced an increased visceromotor response to colorectal distention in awake and anesthetized rats. In pre-WA rats, colorectal distention evoked significant increases in regional blood flow in the cerebellum and periaquaductal gray (PAG). Colorectal distention post-WA revealed activation clusters covering the PAG as well as somatosensory cortex and hippocampus. At maximal colorectal distention, the frontal cortex was significantly deactivated.
\end{abstract}

Address for Correspondence

Mira M. Wouters, Department of Gastroenterology, Translational Research Center for Gastrointestinal Disorders,

Herestraat 49 bus 701,3000 Leuven, Belgium.

Tel: (32) 163308 37; fax: (32) 163459 39;

e-mail: mira.wouters@med.kuleuven.be

Received: 3 January 2012

Accepted for publication: 20 March 2012
Conclusions $\leftrightarrow$ Inferences WA stress induced increased pain perception as well as activation of the somatosensory cortex, PAG, and hippocampus in maternal-separated rats. These findings are in line with human studies and provide indirect evidence that the maternal separation model mimics the cerebral response to visceral hypersensitivity in humans.

Keywords brain mapping, hippocampus, nociception, periaqueductal gray, somatosensory cortex.

\section{INTRODUCTION}

The irritable bowel syndrome (IBS) is the most common functional gastrointestinal disorder, characterized by abdominal pain and altered bowel habits in the absence of an organic cause. ${ }^{1}$ The majority of patients show increased pain sensitivity to balloon distention in the distal gastrointestinal tract, which is considered the main pathophysiological mechanism in IBS. ${ }^{2}$ In addition to peripheral mechanisms (increased excitability of afferent nerve fibers), the so-called visceral hypersensitivity (VHS) may also result from altered processing of visceral pain signals in the brain. ${ }^{3}$ Functional magnetic resonance imaging (fMRI) and positron emission tomography (PET) studies have demonstrated different activation of brain areas involved in emotional, cognitive, and sensory aspects of pain processing (amygdala, insula, and prefrontal cortex) in IBS compared with controls. ${ }^{4-6}$ However, recent data emphasize that brain imaging data are to a large extent influenced by anticipation and associated anxiety as well as comorbid depression and anxiety disorders, complicating the interpretation of the mechanisms involved in altered brain processing. ${ }^{7,8}$ 
Early life events including childhood abuse and neglect are increasingly recognized as major risk factor for the development of depression ${ }^{9}$ and IBS in adulthood. ${ }^{10-12}$ Based on these human data, maternal separation was used to create an animal model of early life stress and a depression-like syndrome. ${ }^{13-15}$ In this model, rat pups are separated from their mother from day 2 to day 14 after birth for several hours per day. Similar to the human situation, this early life stressor alters the response to stress later in life, as demonstrated by the development of VHS after an acute stressor in separated - but not in non-separated-littermates. ${ }^{15,16}$

In rodents, evidence on brain activation in response to painful colorectal distention is mainly based on postmortem assessment of c-fos immunoreactivity, an indirect marker of neuronal activity. ${ }^{17,18}$ One of the limitations of using c-fos as an activation marker is that it provides a mere single time point measurement. In order to obtain a more integrated and dynamic picture of brain activation, in vivo imaging studies such as fMRI or PET scanning are superior, ideally combined with an objective measure of the evoked pain response. Because of the technical difficulties encountered in small animals, only a limited number of studies have been published using imaging tools in rats or mice. ${ }^{19,20}$ Johnson et al. ${ }^{21}$ evaluated brain activation by fMRI during colorectal distention but no data on the pain response were reported, making comparison between different experimental conditions impossible. Therefore, we developed a model in which brain activation by colorectal distention is assessed using $\mathrm{H}_{2}{ }^{15} \mathrm{O}$ microPET imaging in combination with simultaneous recording of the pain response. This setup was designed to evaluate the hypothesis that VHS in maternal separated (MS) rats evoked by an acute stressor is accompanied by altered pain processing in the brain.

\section{MATERIALS AND METHODS}

\begin{abstract}
Animals
Fifteen male Long-Evans rats (Harlan, Horst, The Netherlands) were kept in standard macrolon cages with a layer of wood shavings and housed under conditions of controlled light (06:0018:00 h), temperature $\left(20-22{ }^{\circ} \mathrm{C}\right)$, and humidity $(45 \%)$. Water and food (SDS; Technilab BMI, Someren, The Netherlands) were available ad libitum. All experiments were conducted under a protocol approved by the Institutional Animal Care and Use Committee of the Academic Medical Center (AMC, The Netherlands) and University of Leuven (Leuven, Belgium).
\end{abstract}

\section{Maternal separation}

Pregnant dams reared pups and only male pups were subjected to the maternal separation protocol. During separation, dams were placed in another cage in a separate room for $3 \mathrm{~h} \mathrm{day}^{-1}$ from postnatal day 2 to 14 . In the meantime, the litter was left undisturbed in the cage under infrared light $\left(27-30^{\circ} \mathrm{C}\right)$. Maternal-separated pups were weaned on postnatal day 22 and housed in pairs of two.

\section{Water avoidance protocol}

Acute stress at adult age (400-500 g) was given by water avoidance (WA). In short, rats were positioned on a platform $(8 \times 6 \mathrm{~cm})$ surrounded by water for $1 \mathrm{~h}$. This protocol has been shown to induce enhanced sensitivity to colonic distension in maternalseparated but not in non-handled rats. ${ }^{15}$

\section{Measurement of the visceromotor response to colorectal distension as read-out for visceral pain}

At a minimum age of 6 months, visceral pain was induced by colorectal distention and quantified by the visceromotor response (VMR), i.e., pain-induced abdominal contractions, as previously described. ${ }^{15}$ For colorectal distention, a latex balloon (Ultracover 8F; International Medical Products, Zutphen, The Netherlands) was $5 \mathrm{~cm}$ anally inserted into the descending colon during isoflurane anesthesia. Distensions were performed by inflation of graded volumes of water $(1.0,1.5$, and $2.0 \mathrm{~mL})$. The length and diameter of the balloon during maximum volume distension were 18 and $15 \mathrm{~mm}$, respectively. After each 20-s distension episode, water was quickly removed and a 120-s resting period was allowed. The VMR, a supraspinal reflex generally accepted as the most reliable biomarker to quantify visceral pain in experimental animals, ${ }^{22}$ was measured and quantified by electromyography (EMG). EMG signals were recorded by our validated radio-telemetry setup in the awake rat, $24 \mathrm{~h}$ before the PET scanning, to evaluate visceral sensitivity (Fig. 1). Because the telemetric transmitters failed to record EMG in the PET environment, EMG was quantified by superficial electrodes (for details see below) during brain imaging.

Awake rats We previously validated a radio-telemetry technique in freely moving rats to record pain-induced VMR. As described by Welting et al. ${ }^{15}$ during colorectal distention, animals were placed in a standard macrolon cage (exact size of the receiver) that was positioned on top of the receiver. The receiver (Data Sciences International) was linked through a raw data analog converter (Data Sciences International) to a Biopac MP100 data acquisition system consisting of a converter and specialized software on a personal computer for EMG data storage (Biopac Systems, Inc., Santa Barbara, CA, USA).

Anesthetized rats Because the magnetic field based radio-telemetry system was not compatible with the micro-PET environment, the VMR during brain imaging was quantified in anesthetized rats using superficial neonatal 3M Red Dot Diagnostic ECG electrodes (3M, Diegem, Belgium) positioned at the abdominal musculature. The electrodes were connected to a differential AC amplifier obtained from the A-M Systems Inc. [Cambridge Electronic Design (CED) Limited, Cambridge, UK] and digitized. Data were recorded and analyzed using the sPIKE2 version 6 software from CED Limited.

\section{MicroPET imaging}

Anesthesia Alpha chloralose anesthesia is a mild hypnotic drug that does not produce complete anesthesia allowing us to record 
Protocol 1. Sham, $1,1.5$ and $2 \mathrm{~mL} C R D$ in non stressed $M S$ rats

$n=7$

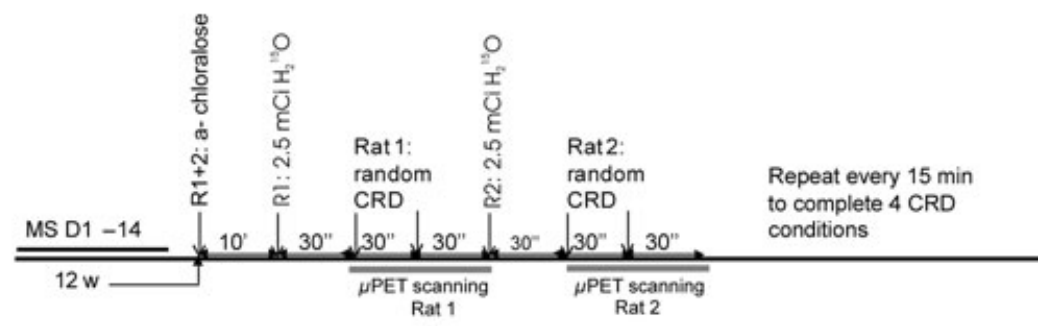

Protocol 2. Visceral perception to $2 \mathrm{~mL} C R D$ pre- versus postWA

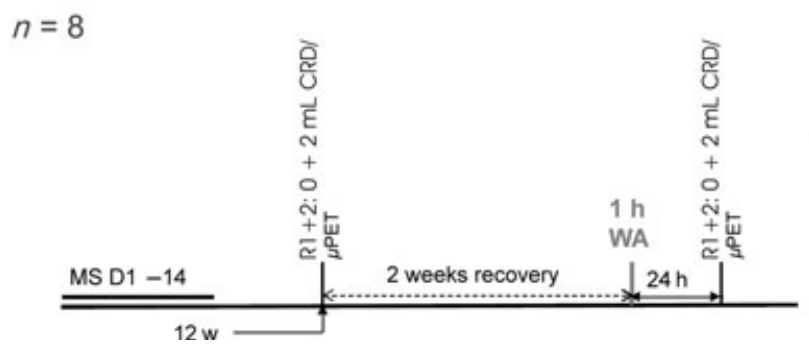

Figure 1 Experimental protocols to register brain activity and visceromotor response simultaneously. Protocol 1: To study the effect of noxious visceral stimuli on regional blood flow and visceromotor response, pre-WA maternal-separated rats underwent colorectal distention (CRD) with various applied distention volumes $(0,1,1.5$, and $2 \mathrm{~mL}$ distention, randomized). Protocol 2: To evaluate the effect of psychological stress on regional blood flow and visceromotor response, rats were scanned pre and post-WA. Rats received only 0 and 2 mL colorectal distention before WA and were allowed to recover for at least 2 weeks. Rats were subjected to water avoidance (WA) and $24 \mathrm{~h}$ later; rats were again subjected to sham and $2 \mathrm{~mL}$ colorectal distention during microPET analysis. Legend: $\mathrm{R} 1+2$ : rats 1 and 2 .

abdominal muscle contraction as read-out for visceral pain. Alpha chloralose anesthesia has been shown to yield reproducible results in repetitive fMRI studies. ${ }^{23}$

During anesthesia with $2-2.5 \%$ isoflurane, a catheter was secured in the tail vein and a bolus alpha chloralose $\left(42.6 \mathrm{mg} \mathrm{kg}^{-1}\right.$ in $<0.5 \mathrm{~mL}$ volume) was injected, followed by a maintenance dose of $22.5 \mathrm{mg} \mathrm{kg}^{-1} \mathrm{~h}^{-1}$ via i.v. infusion.

MicroPET scanning Brain activity was expressed as the relative change in regional cerebral blood flow $(\mathrm{rCBF})$ using $\mathrm{H}_{2}{ }^{15} \mathrm{O}$ uptake as indicator. ${ }^{24}$ Small animal PET imaging was performed using a lutetium oxyorthosilicate detector-based tomography (microPET Focus 220; Siemens Medical Solutions USA, Inc., Malvern, PA, USA), which has a transaxial resolution of $1.35 \mathrm{~mm}$ in full-width at half-maximum. Data were acquired in a $128 \times 128 \times 95$ matrix with a pixel width of $0.475 \mathrm{~mm}$ and a slice thickness of $0.796 \mathrm{~mm}$. The following scanning protocols were used.

Scanning protocol 1. This protocol (Fig. 1) was used to evaluate regional blood flow changes in response to $1,1.5$, and $2 \mathrm{~mL}$ colorectal distention in seven pre-WA rats. The first colorectal distention protocol was applied $10 \mathrm{~min}$ after the isoflurane induction anesthesia was ended. Prior to data acquisition, approximately $2.5 \mathrm{mCi}$ of $\mathrm{H}_{2}{ }^{15} \mathrm{O}$ (range: $1.95-3.21 \mathrm{mCi}$ in $140-$ $650 \mu \mathrm{L}$ ) was injected into the tail vein using an infusion needle set. Acquisitions were started $30 \mathrm{~s}$ after tracer injection. Acquisition data were Fourier rebinned in two frames $(2 \times 30 \mathrm{~s})$, with the colon distended in the first frame. Upon radioactive decay of $\mathrm{H}_{2}{ }^{15} \mathrm{O}$, i.e., after $\sim 15 \mathrm{~min}$ (half-life $=2,1 \mathrm{~min}$ ), the same procedure was repeated for a second, third, and fourth distention volume $(0,1,1.5$, and $2 \mathrm{~mL}$ distention in randomized order to avoid habituation).
Scanning protocol 2. In a second study protocol, the effect of WA on visceral pain perception was evaluated. To decrease the time of anesthesia, the acquisition protocol was shortened and only the sham (balloon inserted, no distention) and $2 \mathrm{~mL}$ stimuli were applied. Eight rats were scanned pre-WA as described in scanning protocol 1. Rats were allowed to recover from the anesthesia for at least 2 weeks. After 2 weeks, rats underwent $1 \mathrm{~h}$ of WA stress, followed by a post-WA PET scanning session after $24 \mathrm{~h}$ (Fig. 1). Rats were killed by an overdose of pentobarbital after the final PET scan.

\section{Functional brain-mapping data analysis}

All sinograms were reconstructed using filtered backprojection. To obtain maximal use of image information without a priori knowledge of brain areas involved, images were analyzed on a voxel-by-voxel basis using SPM2 (Statistical Parametric Mapping, Wellcome Department of Cognitive Neurology, London, UK; http://www.fil.ion.bpmf.ac.uk/spm//. To increase the signalto-noise ratio in the group comparison, we averaged all distention volumes in scanning protocol 1 . Images of different distention volumes were corrected for attenuation and smoothed with an isotropic Gaussian kernel of $2 \times 2 \times 2 \mathrm{~mm}$. Images were corrected for movement by alignment against the average image for an acquisition set and extracerebral activity was masked. Images were spatially normalized to Paxinos space, ${ }^{25}$ as previously described. ${ }^{26}$

\section{Statistics}

Visceromotor response Using the telemetric device in awake rats or the superficial electrodes in anesthetized rats, area under curve 
or the number of spikes per second of distention vs response (normalized data sets) were used, respectively, to calculate differences between groups. Statistics were performed by a two-way ANOVA test with Bonferroni posttests.

Brain imaging All brain imaging analyses were performed at group level in a multifactor design using subjects $\times$ conditions. For a voxel-based correlation analysis between $\mathrm{H}_{2}{ }^{15} \mathrm{O}$ uptake and distention volume, a single subject design with covariates only was used. A statistical threshold of Puncorrected $_{\text {voxel }}<0.01$ (uncorrected for multiple comparisons) was used together with a cluster extent threshold $k_{\mathrm{E}}>100$ voxels.

\section{RESULTS}

\section{Visceral pain response to noxious colorectal distention}

Colorectal distention resulted reproducibly in a volume-dependent increase in EMG response in maternalseparated rats (up to $4 \mathrm{~h}$ under anesthesia, data not shown). To evaluate whether PET imaging itself would represent a stressor and thus induce VHS, VMR to colorectal distention $(1,1.5$, and $2 \mathrm{~mL})$ was evaluated in a pilot set of maternal-separated rats $(n=3)$ before and after the initial PET scan. Colorectal distention applied 12 days after PET scanning did not increase VMR, indicating that this procedure is not stressful enough to induce VHS in these animals (data not shown). In contrast, WA stress resulted in an increased VMR response in awake rats (protocol 1, $n=7$, $41.7 \pm 10.71 \%$ increase in VMR at $2 \mathrm{~mL}$ distention, $P<0.0001$; Fig. 2A) as well as in anesthetized rats (protocol 2, $n=6$, pre-WA $0.45 \pm 014$ vs post-WA $1.24 \pm 0.24$ spikes per second, $P<0.05$; Fig. $2 B$ ), confirming the validity of the WA protocol to induce VHS.

\section{Protocol 1. Brain responses to colorectal distention in maternal-separated rats}

In the analysis at group level, we compared the average image of $1,1.5$, and $2 \mathrm{~mL}$ distention volume to the sham (balloon inserted, no distention) image. Comparing colorectal distention with sham animals $(n=7)$ yielded significant activation of one large cluster covering the periaqueductal gray (PAG; Fig. 3). Comparison of sham with distention yielded significant deactivation in the frontal cortex and caudate putamen (Fig. 3).

Performing correlation analyses on the images acquired during the various distention volumes revealed that activation of the PAG was not dependent on the distention volume or visceromotor response (Fig. 4A,B). Deactivation of the right frontal cortex was
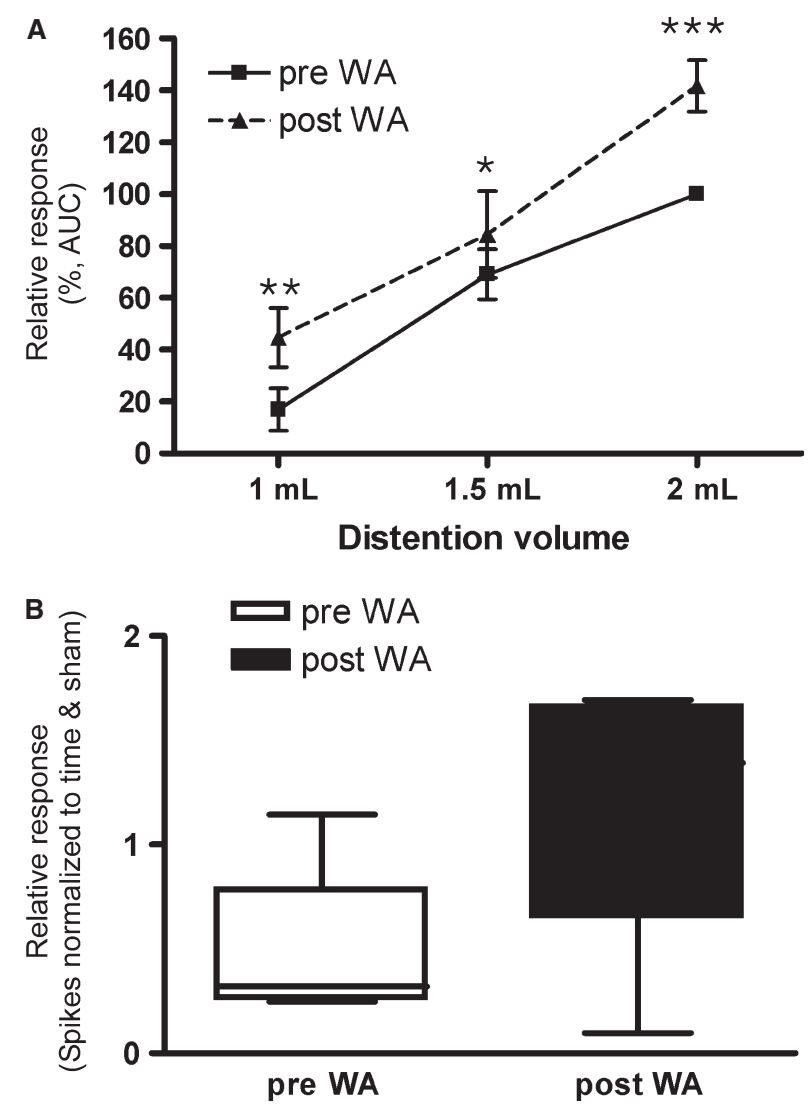

Figure 2 Effect of increasing distention volume on the visceromotor response in awake maternal-separated rats before and after water avoidance $\left(\mathrm{A}, n=7,{ }^{\star \star} P<0.0001\right.$ by a two-way ANOvA test with Bonferroni posttest). Data were normalized to the value obtained during the maximum $2 \mathrm{~mL}$ distention volume pre-WA. (B) Visceromotor response triggered by $2 \mathrm{~mL}$ colorectal distention during PET scanning before and after WA $(n=6, P<0.05)$ in anesthetized rats. The horizontal line indicates the median value and the lower and upper brackets indicate the 25 th percentile interquartile range and 75 th percentile, respectively.

negatively correlated $\left(r^{2}=0.95\right)$ with the visceromotor response (Fig. $4 \mathrm{C}, \mathrm{D})$, with a maximal deactivation of $12 \%$ at $2 \mathrm{~mL}$ distention.

Detailed cluster peak locations and $P$-values of SPM analyses during colorectal distention pre-WA are shown in Table 1.

\section{Protocol 2. Effect of acute WA on brain responses to colorectal distention in maternal-separated rats}

In a second study protocol, brain activation evoked by $2 \mathrm{~mL}$ colorectal distention compared with sham was evaluated before and $24 \mathrm{~h}$ after WA to identify the brain areas involved in VHS. Similar to awake rats, WA induced increased pain perception to $2 \mathrm{~mL}$ colorectal distention in anesthetized rats (Fig. 2B). 


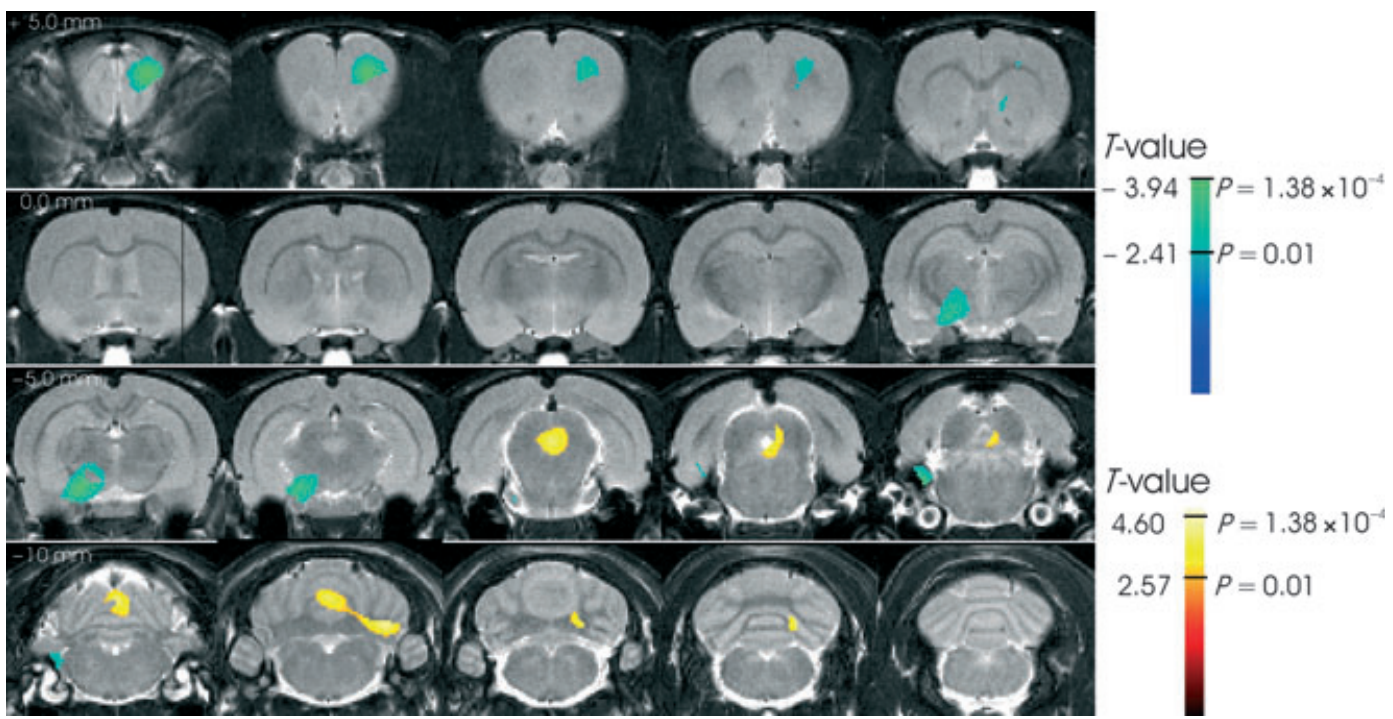

Figure 3 Categorical comparison of $\mathrm{H}_{2}{ }^{15} \mathrm{O}$ cerebral blood flow in coronal brain sections at basal level and during distention showing significant activation in yellow and deactivation in green-blue $\left(n=7\right.$; figure given at $P_{\text {height }}<0.01$, uncorrected). Significance is shown with a $t$-statistic color scale, which corresponds to the level of significance at the voxel level. The distance between the sections is $1.0 \mathrm{~mm}$ with the position relative to Bregma on top of the sections.

\section{Specific brain nuclei activated by colorectal distention}

When comparing $2 \mathrm{~mL}$ colorectal distention $v s$ sham before WA $(n=8)$, colorectal distention $(2 \mathrm{~mL})$ evoked significant activation of the area surrounding the PAG (Table 2). Twenty-four hours after WA, in addition to the PAG area, the hippocampus and primary somatosensory cortex were significantly activated, associated with the development of VHS. Comparison of the group analyses post vs pre-WA confirmed significant activation of the primary somatosensory cortex in post- vs pre-WA maternalseparated rats (Table 2).

\section{Specific brain nuclei deactivated by colorectal distention}

Before WA, comparison of sham vs colorectal distention revealed no deactivated brain areas by $2 \mathrm{~mL}$ colorectal distention. In contrast, after WA, i.e., in visceral hypersensitive rats, the frontal cortex was deactivated (Table 2). Comparing of prevs post-WA confirmed more deactivation of the frontal cortex in post-WA rats compared with pre-WA.

Detailed cluster peak locations and P-values of SPM analyses during colorectal distention in pre-WA normo- VS post-WA hypersensitive rats are provided in Table 2.

\section{DISCUSSION}

Early life stress by neonatal MS predisposes to the development of VHS ${ }^{15,16,27}$ but the mechanisms underlying VHS are not well understood. Using water PET imaging, we here demonstrated for the first time that the development of VHS in maternal-separated rats is associated with activation of the PAG, hippocampus, and primary somatosensory cortex, as well as deactivation of the frontal cortex. These results demonstrate that altered brain processing of incoming nociceptive signals may represent an important pathophysiological mechanism leading to VHS and indicate that the used maternal-separated model described may be of great interest to gain more insight in the central pathways and neurochemical mechanisms involved.

Colorectal distention in pre- and post-WA maternalseparated rats revealed activation of a large cluster covering the dorsal pons/PAG, a region involved in antinociception. ${ }^{28}$ The PAG modulates the descending inhibitory pathway and is known to exhibit analgesic effects through opiate and glutamate NMDA receptor activation. $^{29}$ Mayer et $^{2}$. $^{6}$ hypothesized that IBS patients fail to appropriately activate an inhibitory corticolimbic system associated with effective pain inhibition by the PAG. In our maternal separation rat model, activation of the PAG was not dependent on the pain stimulus intensity as reflected by VMR. PAG activation in the post-WA, hypersensitive rats had the same intensity as in the pre-WA rats, suggesting no 
A
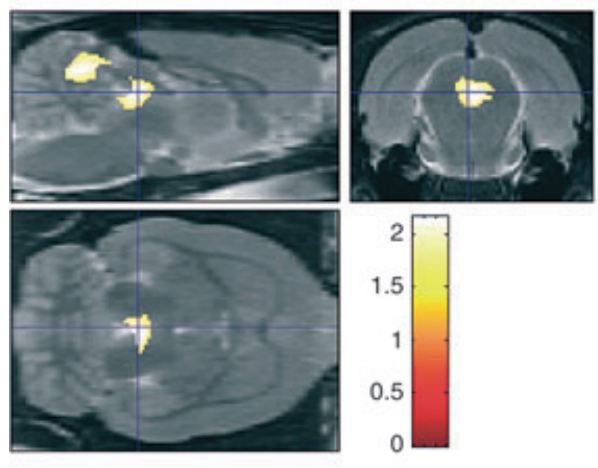

C

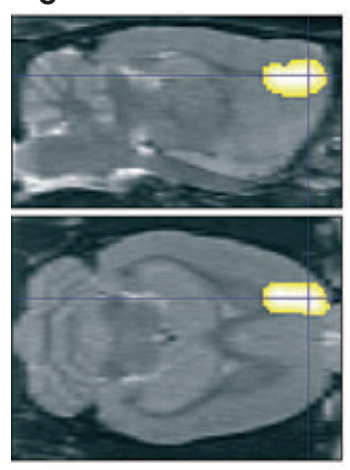

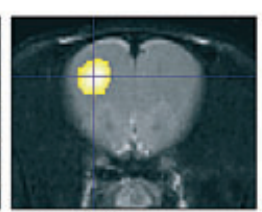

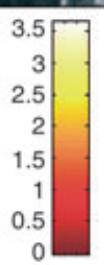

B
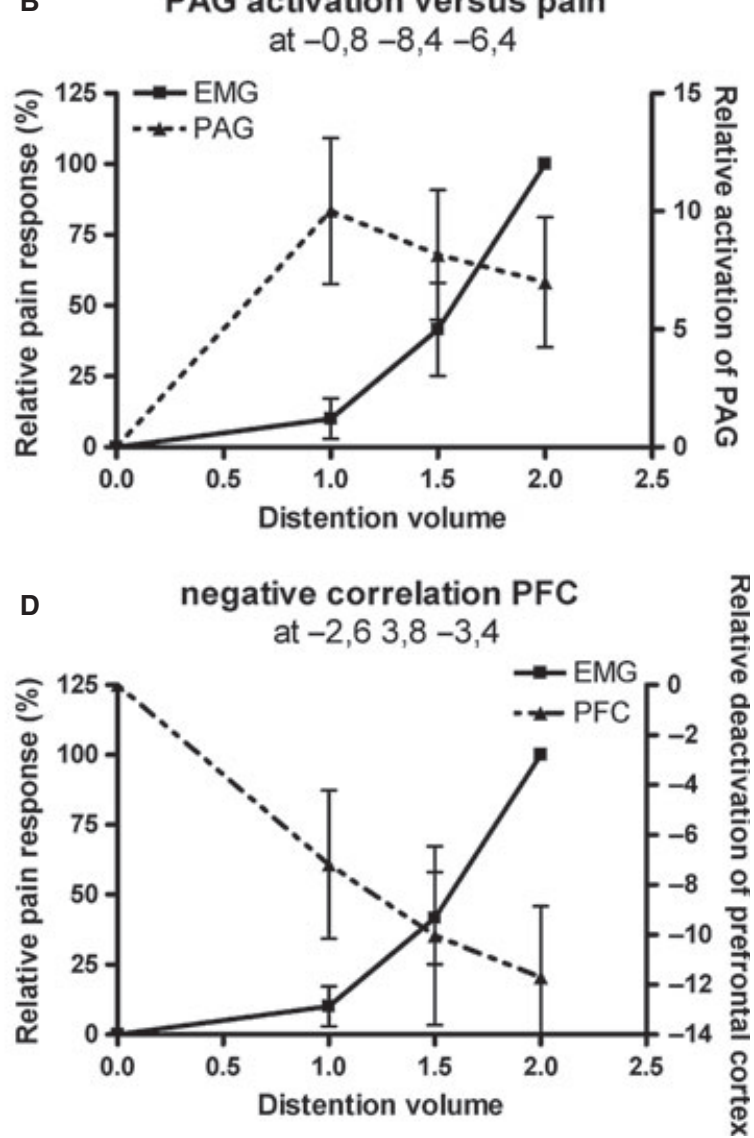

Figure 4 Changes in regional cerebral blood flow and visceromotor response in response to colorectal distention. Colorectal distention in pre-WA maternal-separated rats resulted in activation of the area covering the periaquaductal gray (PAG, Fig. 3A.) and the cerebellum. This effect did not correlate with the VMR (Fig. 3B.). In contrast, colorectal distention induced deactivation of the frontal cortex (Fig. 3C.), which negatively correlated with the visceromotor response (Fig. $3 \mathrm{D}$, slope correlation $\left.=-6.24 ; r^{2}=0.9482\right) . n=7$, data are expressed as mean \pm SEM.

differences in endogenous pain inhibition. In line with human data, ${ }^{30}$ maternal-separated rats also showed deactivation of the medial prefrontal cortex, which was more pronounced in the post-WA hypersensitive rats than in the pre-WA rats, suggesting that VHS in a model of maternal separated is due to altered pain modulation by the prefrontal cortex.

Besides the PAG region, colorectal distention in postWA rats showed activation of the hippocampus and somatosensory cortex, indicating more activation of higher brain areas than in pre-WA rats. Alterations in hippocampus activation and corticotropin-releasing factor regulation may affect HPA axis regulation, contributing to behavioral changes associated with stress-related disorders and altered visceral pain modulation. $^{31,32}$ Additionally, in functional dyspepsia patients, the subgroup characterized by a history of abuse shows abnormal hippocampus/amygdala responses to visceral pain. ${ }^{33}$ This finding thus adds proof to the validity of our model to mimic the human condition.
The somatosensory cortex on the other hand is a complex system integrating incoming sensory modalities including nociception. Activation of the somatosensory cortex in post-WA but not in pre-WA rats clearly demonstrates higher visceral afferent input to the brain. Rosenberger et al. ${ }^{29}$ reported on the effect of acute stress by increasing anxiety levels and evoking activation of the somatosensory cortex by colorectal distention. These results clearly suggest that in maternal-separated rats, parallel to humans, an anxious psychological trait potentiates visceral pain by activating the somatosensory cortex.

Visceral pain processing is complex and starts with sensing by nociceptors present on peripheral afferent nerve fibers. This nociceptive signal travels via the spinal cord to the brain where it is processed and consciously perceived at the cortical level. Alterations in any of these steps including upregulation of peripheral nociceptors can result in increased visceral pain perception. Recent human studies demonstrated 
Table 1 Peak locations for the clusters in the group comparison and correlation analysis of protocol 1 (at $P_{\text {height }}<0.01$ uncorrected, $k_{\mathrm{E}}>100$ )

\begin{tabular}{|c|c|c|c|c|c|c|c|c|}
\hline & \multicolumn{2}{|c|}{ Cluster level } & \multicolumn{2}{|c|}{ Voxel level } & \multicolumn{3}{|c|}{ Structure } & \multirow[b]{2}{*}{ Name } \\
\hline & $k_{\mathrm{E}}$ & $P_{\text {uncorr }}$ & $t$ & $P_{\text {uncorr }}$ & $x$ & y & $z$ & \\
\hline \multirow[t]{4}{*}{ Average dist $>$ sham } & 1725 & 0.136 & 3.35 & 0.001 & -0.2 & -10.6 & -3.4 & Cerebellum \\
\hline & & & 3.27 & 0.001 & -4.2 & -11.4 & -5.8 & \\
\hline & & & 3.21 & 0.001 & -2.2 & -12.8 & -5 & \\
\hline & & & 3.15 & 0.001 & -0.2 & -7.2 & -4.8 & Periaqueductal gray \\
\hline \multirow[t]{4}{*}{ Sham $>$ average dist } & 1708 & 0.138 & 3.94 & 0 & -2.2 & 4.6 & -3.8 & Frontal cortex \\
\hline & & & 2.73 & 0.005 & -2.6 & 1.8 & -3.2 & Dorsal striatum \\
\hline & & & 2.67 & 0.005 & -1.6 & 1.4 & -5.6 & \\
\hline & 1704 & 0.138 & 3.59 & 0 & 2.6 & -5.2 & -8 & Pons \\
\hline
\end{tabular}

Negative correlation PFC with distention volume $\left(\right.$ at $P_{\text {height }}<0.01$ uncorrected, $\left.k_{\mathrm{E}}>100\right)$

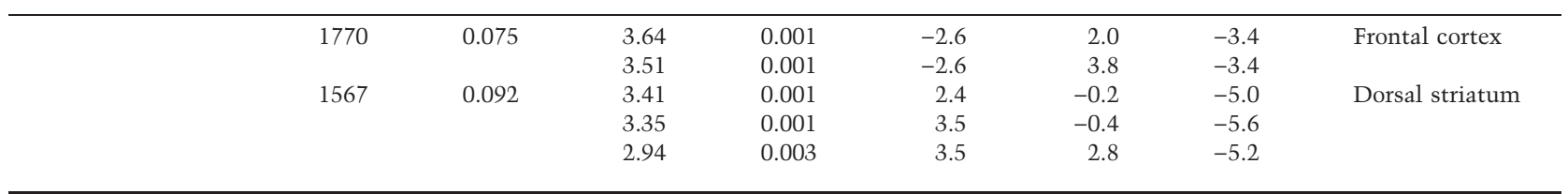

Dist, distention. $P_{\text {uncorr }}$ at cluster level: the chance $(\mathrm{p})$ of finding a cluster with this or a greater size $\left(k_{\mathrm{E}}\right)$, uncorrected for search volume, $k_{\mathrm{E}}=$ cluster extent, $t=$ measure of the statistical significance. $P_{\text {uncorr }}$ at voxel level: the chance (p) of finding (under the null hypothesis) a voxel with this or a greater height ( $t$-statistic), uncorrected for search volume, $x=$ lateral distance in $\mathrm{mm}$ from the midline (negative values to the right side), $y=$ anteroposterior location relative to Bregma (negative values: posterior to Bregma), $z=$ dorsoventral position (based upon the Paxinos stereotactic atlas).

Table 2 Peak locations for the clusters in the group comparison of protocol 2 (at $P_{\text {height }}<0.01$ uncorrected, $k_{\mathrm{E}}>100$ )

\begin{tabular}{|c|c|c|c|c|c|c|c|c|}
\hline & \multicolumn{2}{|c|}{ Cluster level } & \multicolumn{2}{|c|}{ Voxel level } & \multicolumn{3}{|c|}{ Structure } & \multirow[b]{2}{*}{ Name } \\
\hline & $k_{\mathrm{E}}$ & $P_{\text {uncorr }}$ & $t$ & $P_{\text {uncorr }}$ & $x$ & y & $z$ & \\
\hline \multicolumn{9}{|l|}{ Activation } \\
\hline $2 \mathrm{~mL}$ Dist $>$ sham pre-WA & 902 & 0.289 & 3.62 & 0.001 & -0.8 & -8.4 & -6.4 & Periaqueductal gray \\
\hline \multirow{4}{*}{$2 \mathrm{~mL}$ Dist $>$ sham post-WA } & 1378 & 0.193 & 4.58 & 0 & -0.4 & -7.8 & -6.4 & Periaqueductal gray \\
\hline & 285 & 0.562 & 2.95 & 0.004 & 4.0 & -5.8 & -3.6 & Hippocampus \\
\hline & 116 & 0.729 & 2.93 & 0.005 & 4.0 & 1.8 & -1.4 & Primary somatosensory cortex \\
\hline & 104 & 0.745 & 2.89 & 0.005 & 0.2 & 2.8 & -5.4 & Infralimbic cortex (=medial PFC) \\
\hline (Dist > sham post-WA)-(Dist > sham pre-WA) & 470 & 0.448 & 3.37 & 0.002 & 4 & 1.4 & -1.4 & Primary somatosensory cortex \\
\hline \multicolumn{9}{|l|}{ Deactivation } \\
\hline \multicolumn{9}{|l|}{ No significant deactivation pre-WA } \\
\hline $2 \mathrm{~mL}$ Dist $<$ sham post-WA & 148 & 0.689 & 2.78 & 0.002 & 0.8 & -0.8 & -9.4 & Frontal cortex \\
\hline (Sham post-WA > dist $)-($ Sham pre-WA $>$ dist $)$ & 689 & 0.355 & 2.96 & 0.004 & -1.4 & 1.2 & -8.4 & Frontal cortex \\
\hline
\end{tabular}

Dist, distention. $P_{\text {uncorr }}$ at cluster level: the chance $(\mathrm{p})$ of finding a cluster with this or a greater size $\left(k_{\mathrm{E}}\right)$, uncorrected for search volume, $k_{\mathrm{E}}=$ cluster extent, $t=$ measure of the statistical significance. $P_{\text {uncorr }}$ at voxel level: the chance $(\mathrm{p}$ ) of finding (under the null hypothesis) a voxel with this or a greater height ( $t$-statistic), uncorrected for search volume. $x=$ lateral distance in $\mathrm{mm}$ from the midline (negative values to the right side). $y=$ anteroposterior location relative to Bregma (negative values: posterior to Bregma). $z=$ dorsoventral position (based upon the Paxinos stereotactic atlas).

altered brain activity in response to visceral stimuli in IBS, ${ }^{4,5,7,29}$ suggesting that central mechanisms contribute to abnormal pain perception. Although reports on brain imaging in IBS patients are heterogeneous, several studies report activation of the insula, cingulate cortex, and prefrontal cortex subregions by rectal distentions in IBS patients. ${ }^{4,5,7}$ However, the activation of insula and cingulate by colorectal distention as reported in human studies may be more related to cognitive/affective aspects of the subjective (human) pain experience - which are more limited in rodents and are abolished by anesthesia - than with VHS itself.
Moreover, given the frequent psychopathology in IBS personality traits such as neuroticism and state psychological factors such as anxiety not only aggravate but also trigger IBS symptoms - it is crucial to take into account that such affective disturbances may contribute to disturbed neural responses to visceral stimuli in IBS. Along the same line, Elsenbruch et al. reported that anxiety symptoms correlated with pain-induced activation of the cingulate cortex whereas depression correlated with prefrontral and cerebellar activation during visceral pain in IBS. ${ }^{7,8}$ Moreover, group differences in pain-induced activation between IBS patients 
and healthy controls largely disappeared when controlling for comorbid anxiety and depression. In parallel, Simmons et al. ${ }^{34}$ reported that anxiety is related to greater anticipatory reactivity in the brain by activation of the insular and cingulate cortex even in the absence of visceral stimulation. These data seem to indicate that in humans' anxiety and anticipation are the main triggers for activation of the insular and cingulate cortex, areas known to be involved in cognitive functions and anticipation. ${ }^{35}$ As the maternal-separated rats were anesthetized, these confounding factors were lacking, possibly explaining the finding that no activation of the insular and cingulate cortex was detected. Similar results were previously reported by Gibney et al. who reported lack of activation of the cingulate or insula, assessed by c-fos immunohistochemistry, in the spontaneous visceral hypersensitive Wistar-Kyoto compared with normosensitive Sprague-Dawley rats. ${ }^{17}$ The fact that we did observe brain activation in other regions and pain responses suggests that our model provides an interesting model devoid of confounding anticipation processes, to study pain processing in the brain.

We have shown for the first time that $\mathrm{H}_{2}{ }^{15} \mathrm{O}$ PET imaging is feasible and generates reproducible results in rats. As the sensitivity of water PET imaging is reduced compared with human studies because of relative poorer spatial resolution and the relatively large positron range of ${ }^{15} \mathrm{O}$ and microPET resolution, we improved sensitivity by averaging three distention volume images (pre-WA study, 1, 1.5, and $2 \mathrm{~mL}$ distention). Apart from spatial resolution, also the temporal resolution of brain responses acquired with $\mathrm{H}_{2}{ }^{15} \mathrm{O}$ PET is relatively limited. Data were acquired over a 60-s period to achieve an acceptable signalto-noise ratio. This period covers $30 \mathrm{~s}$ of distention and 30 s rest after deflation of the balloon, possibly diluting the signal evoked by noxious colonic distention only. This average results in convolution of multiple neural processes (alerting, deflation, etc.) where information on some brain regions of interest may have been lost. Another study limitation is that we cannot conclude whether the changes in brain activation are causal to increased pain perception or rather reflect increased colorectal signaling to the brain.

In summary, we were able to demonstrate changes in $\mathrm{rCBF}$ in relation to the VMR to colorectal distention in a rat model of maternal separation. Electromyography in pre- $V S$ post-WA rats showed an increased VMR to colorectal distention in both awake and anesthetized rats. Pre-WA rats showed activation in regions involved in antinociception (dorsal pons/PAG) and deactivation in the prefrontal cortex, another pain modulating area. After stress (WA), rats became visceral hypersensitive to colorectal distention, which induced activation of PAG, hippocampus, and somatosensory cortex concomitantly with increased deactivation of the prefrontal cortex. Based on these results, we hypothesize that VHS induced by stress in maternalseparated rats is not because of altered descending pain inhibition by the PAG, but rather results from altered pain modulation at the cerebral level in the somatosensory cortex, hippocampus, and prefrontal cortex. Future experiments using PET radioligand-binding studies of, e.g., serotonergic, cannabinoid, ${ }^{36}$ opioid, $\mathrm{NK}$, and glutamate ${ }^{37}$ system in the rat model of maternal separation may shed light on the mediators involved in the development of VHS.

\section{ACKNOWLEDGMENTS}

The authors like to thank Peter Vermaelen and Ann Van Santvoort for technical assistance with the PET scanning, Kathleen Lambaerts and the radiopharmacy department for their help with the $\mathrm{H}_{2}{ }^{15} \mathrm{O}$ water production, and Olaf Welting for animal care taking.

\section{FUNDING}

Mira Wouters, Cindy Casteels and Lukas Van Oudenhove are financially supported by a Fonds Wetenschappelijk Onderzoek (FWO) postdoctoral grant. Koen Van Laere is a senior clinical investigator of the Flemish Fund for Scientific Research. Guy Boeckxstaens received research funding by a grant from the Flemish government (Odysseus Program, FWO).

\section{CONFLICTS OF INTEREST}

No conflicts of interest for any of the authors.

\section{REFERENCES}

1 Longstreth GF, Thompson WG, Chey WD, Houghton LA, Mearin F, Spiller RC. Functional bowel disorders. Gastroenterology 2006; 130: 1480-91.

2 Mertz H, Naliboff B, Munakata J, Niazi $\mathrm{N}$, Mayer EA. Altered rectal percep- tion is a biological marker of patients with irritable bowel syndrome. Gastroenterology 1995; 109: 40-52.

3 Gebhart GF. Pathobiology of visceral pain: molecular mechanisms and therapeutic implications: IV. Visceral afferent contributions to the pathobiology of visceral pain. Am I Physiol
Gastrointest Liver Physiol 2000; 278 : G834-8.

4 Tillisch K, Labus JS. Advances in imaging the brain-gut axis: functional gastrointestinal disorders. Gastroenterology 2011; 140: 407-11.

5 Rapps N, van Oudenhove L, Enck P, Aziz Q. Brain imaging of visceral 
functions in healthy volunteers and IBS patients. I Psychosom Res 2008; 64: 599-604.

6 Mayer EA, Berman S, Suyenobu B et al. Differences in brain responses to visceral pain between patients with irritable bowel syndrome and ulcerative colitis. Pain 2005; 115: 398-409.

7 Elsenbruch S, Rosenberger C, Bingel U, Forsting $M$, Schedlowski M, Gizewski ER. Patients with irritable bowel syndrome have altered emotional modulation of neural responses to visceral stimuli. Gastroenterology 2010; 139: 1310-9.

8 Elsenbruch S, Rosenberger C, Enck P, Forsting M, Schedlowski M, Gizewski ER. Affective disturbances modulate the neural processing of visceral pain stimuli in irritable bowel syndrome: an fMRI study. Gut 2009; 59: 489-95.

9 McEwen BS. Glucocorticoids, depression, and mood disorders: structural remodeling in the brain. Metabolism 2005; 54: 20-3.

10 Oakley Browne MA, Joyce PR, Wells JE, Bushnell JA, Hornblow AR. Disruptions in childhood parental care as risk factors for major depression in adult women. Aust N Z J Psychiatry 1995; 29: 437-48.

11 Heim C, Nemeroff CB. The role of childhood trauma in the neurobiology of mood and anxiety disorders: preclinical and clinical studies. Biol Psychiatry 2001; 49: 1023-39.

12 Drossman DA. Abuse, trauma, and GI illness: is there a link? Am J Gastroenterol 2011; 106: 14-25.

13 Oitzl MS, Workel JO, Fluttert M, Frosch F, De Kloet ER. Maternal deprivation affects behaviour from youth to senescence: amplification of individual differences in spatial learning and memory in senescent Brown Norway rats. Eur I Neurosci 2000; 12: 3771-80.

14 Sanchez MM, Ladd CO, Plotsky PM. Early adverse experience as a developmental risk factor for later psychopathology: evidence from rodent and primate models. Dev Psychopathol 2001; 13: 419-49.

15 Welting O, van den Wijngaard RM, de Jonge WJ, Holman R, Boeckxstaens GE. Assessment of visceral sensitivity using radio telemetry in a rat model of maternal separation. Neurogastroenterol Motil 2005; 17: 838-45.
16 Coutinho SV, Plotsky PM, Sablad M et al. Neonatal maternal separation alters stress-induced responses to viscerosomatic nociceptive stimuli in rat. Am I Physiol Gastrointest Liver Physiol 2002; 282: G307-16.

17 Gibney SM, Gosselin RD, Dinan TG, Cryan JF. Colorectal distensioninduced prefrontal cortex activation in the Wistar-Kyoto rat: implications for irritable bowel syndrome. Neuroscience 2010; 165: 675-83.

18 Chung EK, Zhang $\mathrm{X}$, Li Z, Zhang $\mathrm{H}$, $\mathrm{Xu} \mathrm{H}$, Bian Z. Neonatal maternal separation enhances central sensitivity to noxious colorectal distention in rat. Brain Res 2007; 1153: 68-77.

19 Lazovic J, Wrzos HF, Yang QX et al. Regional activation in the rat brain during visceral stimulation detected by c-fos expression and fMRI. Neurogastroenterol Motil 2005; 17: 548-56.

20 Wang Z, Bradesi S, Maarek JM et al. Regional brain activation in conscious, nonrestrained rats in response to noxious visceral stimulation. Pain 2008; 138: 233-43.

21 Johnson AC, Myers B, lazovic J, Towner R, Greenwood-van MB. Brain activation in response to visceral stimulation in rats with amygdala implants of corticosterone: an FMRI study. PLOS ONE 2010; 5: e8573.

22 Christianson JA, Gebhart GF. Assessment of colon sensitivity by luminal distension in mice. Nat Protoc 2007; 2: 2624-31.

23 Alonso BC, Makarova T, Hess A. On the use of alpha-chloralose for repeated BOLD fMRI measurements in rats. J Neurosci Methods 2011; 195: 236-40.

24 Fox PT, Mintun MA, Raichle ME, Miezin FM, Allman JM, Van Essen DC. Mapping human visual cortex with positron emission tomography. Nature 1986; 323: 806-9.

25 Schweinhardt P, Fransson P, Olson L, Spenger C, Andersson JL. A template for spatial normalisation of MR images of the rat brain. I Neurosci Methods 2003; 129: 105-13.

26 Casteels C, Vermaelen P, Nuyts J et al. Construction and evaluation of multitracer small-animal PET probabilistic atlases for voxel-based functional mapping of the rat brain. $J \mathrm{Nucl}$ Med 2006; 47: 1858-66.

27 Barreau F, Ferrier L, Fioramonti J, Bueno L. Neonatal maternal deprivation triggers long term alterations in colonic epithelial barrier and mucosal immunity in rats. Gut 2004; 53: 5016.

28 Petrovic P, Ingvar M. Imaging cognitive modulation of pain processing. Pain 2002; 95: 1-5.

29 Rosenberger C, Elsenbruch S, Scholle A et al. Effects of psychological stress on the cerebral processing of visceral stimuli in healthy women. Neurogastroenterol Motil 2009; 21: 740-e45.

30 Van Oudenhove L, Vandenberghe J, Dupont $\mathrm{P}$ et al. Cortical deactivations during gastric fundus distension in health: visceral pain-specific response or attenuation of 'default mode' brain function? A H2 15O-PET study. Neurogastroenterol Motil 2009; 21: 259-71.

31 Bremner JD, Randall P, Vermetten E et al. Magnetic resonance imagingbased measurement of hippocampal volume in posttraumatic stress disorder related to childhood physical and sexual abuse - a preliminary report. Biol Psychiatry 1997; 41: 23-32.

32 Bremner JD, Vythilingam M, Vermetten E et al. MRI and PET study of deficits in hippocampal structure and function in women with childhood sexual abuse and posttraumatic stress disorder. Am I Psychiatry 2003; 160: 924-32.

33 Van Oudenhove L, Vandenberghe J, Dupont $\mathrm{P}$ et al. Regional brain activity in functional dyspepsia: a $\mathrm{H}(2)(15)$ O-PET study on the role of gastric sensitivity and abuse history. Gastroenterology 2010; 139: 36-47.

34 Simmons AN, Stein MB, Strigo IA, Arce E, Hitchcock C, Paulus MP. Anxiety positive subjects show altered processing in the anterior insula during anticipation of negative stimuli. Hum Brain Mapp 2011; 32(11): 1836-46.

35 Naliboff BD, Mayer EA. Brain imaging in IBS: drawing the line between cognitive and non-cognitive processes. Gastroenterology 2006; 130: 267-70.

36 Burns HD, van LK, Sanabria-Bohorquez S et al. [18F]MK-9470, a positron emission tomography (PET) tracer for in vivo human PET brain imaging of the cannabinoid-1 receptor. Proc Natl Acad Sci U S A 2007; 104: 9800-5.

37 Niddam DM, Tsai SY, Lu CL, Ko CW, Hsieh JC. Reduced hippocampal glutamate-glutamine levels in irritable bowel syndrome: preliminary findings using magnetic resonance spectroscopy. Am I Gastroenterol 2011; 106: $1503-11$. 